\title{
Diacronie
}

Studi di Storia Contemporanea

$\mathrm{N}^{\circ} 29,1$ | 2017

"Crash test"

\section{Wikipedia è poco affidabile? La colpa è anche degli esperti}

\section{Cristian Cenci}

\section{(2) OpenEdition}

\section{Journals}

\section{Edizione digitale}

URL: http://journals.openedition.org/diacronie/5445

DOI: $10.4000 /$ diacronie. 5445

ISSN: 2038-0925

\section{Editore}

Association culturelle Diacronie

\section{Notizia bibliografica digitale}

Cristian Cenci, « Wikipedia è poco affidabile? La colpa è anche degli esperti », Diacronie [Online], № 29, 1 | 2017, documento 10, Messo online il 29 mars 2017, consultato il 01 mai 2019. URL : http:// journals.openedition.org/diacronie/5445; DOI : 10.4000/diacronie.5445 


\title{
Diacronie
}

Studi di Storia Contemporanea

29, 1/2017

"Crash test". Continuità, discontinuità, legami e rotture nelle dinamiche della storia contemporanea

\section{Wikipedia è poco affidabile? La colpa è anche degli esperti}

\author{
Cristian CENCI
}

Per citare questo articolo:

CENCI, Cristian, «Wikipedia è poco affidabile? La colpa è anche degli esperti», Diacronie. Studi di Storia Contemporanea "Crash test". Continuità, discontinuità, legami e rotture nelle dinamiche della storia contemporanea, 29, 1/2017, 29/3/2017,

URL: <http://www.studistorici.com/2017/03/29/cenci_numero_29/ >

Diacronie Studi di Storia Contemporanea $\rightarrow$ http://www.diacronie.it

Rivista storica online. Uscita trimestrale.

redazione.diacronie@hotmail.it

Comitato di direzione: Naor Ben-Yehoyada - João Fábio Bertonha - Christopher Denis-Delacour - Maximiliano Fuentes Codera Anders Granås Kjøstvedt - John Paul Newman - Deborah Paci - Niccolò Pianciola - Spyridon Ploumidis - Wilko Graf Von Hardenberg

Comitato di redazione: Jacopo Bassi - Luca Bufarale - Gianluca Canè - Fausto Pietrancosta - Alessandro Salvador - Matteo Tomasoni Diritti: gli articoli di Diacronie. Studi di Storia Contemporanea sono pubblicati sotto licenza Creative Commons 3.0. Possono essere riprodotti e modificati a patto di indicare eventuali modifiche dei contenuti, di riconoscere la paternità dell'opera e di condividerla allo stesso modo. La citazione di estratti è comunque sempre autorizzata, nei limiti previsti dalla legge. 


\title{
10/ Wikipedia è poco affidabile? La colpa è anche degli esperti
}

\author{
Cristian CENCI
}

Quale rapporto si sta costruendo fra le scienze storiche e Wikipedia? Come bisogna porsi di fronte alle voci di Wikipedia che trattano di storia? Quale potrà essere il ruolo della futura "enciclopedia libera" per lo studio e l'insegnamento della storia? A partire dall'analisi di alcune voci di Wikipedia operata dal gruppo di lavoro "Nicoletta Bourbaki", sei autori animano una tavola rotonda sul rapporto tra l'enciclopedia libera e il mondo della storia.

Scrivere di storia su Wikipedia è un processo stimolante e interessante. Per affrontarlo, però, è necessario armarsi di molta pazienza perché Wikipedia è sicuramente un progetto con un nobile scopo ma che non scampa ad alcune delle dinamiche più frequenti del web. Anzi, occupandosi di cultura ed essendo per sua natura un progetto collaborativo, essa può diventare un'arena di scontro ideologico quando si inizia a trattare di argomenti sensibili, come possono essere quelli a sfondo storico.

La comunità di Wikipedia in italiano può contare in media su un migliaio di utenti attivi ${ }^{1}$, cioè utenti registrati che hanno effettuato almeno qualche modifica nell'ultimo mese, ma questo numero ridotto di fatto limita l'ampiezza e la competenza che è possibile raggiungere in un'enciclopedia che tende ad abbracciare moltissime branche della conoscenza umana.

Le voci di alcuni argomenti di storia sono sicuramente tra quelle che stuzzicano il maggiore interesse tra i wikipediani, ed infatti alcuni temi vantano un'ampiezza ed un livello di approfondimento notevole. Basta, ad esempio, soffermarsi sulle voci dei due conflitti mondiali per notare il grande numero di articoli correlati che trattano finanche di battaglie e situazioni minori delle quali il pubblico generalista non ha mai nemmeno sentito parlare. Ed è proprio il target di pubblico che per primo differenzia l'enciclopedia online dalle riviste specialistiche: Wikipedia è il

\footnotetext{
${ }^{1}$ Statistiche di Wikipedia, URL: < https://stats.wikimedia.org/IT/Sitemap.htm > [consultato il 7 febbraio 2017].
} 
sesto sito più visitato al mondo ${ }^{2} \mathrm{e}$ in Italia $^{3}$, e come tale è la prima fonte di informazioni strutturate ad essere consultata dai fruitori del web. Come rendere, quindi, il più competente possibile l'analisi storica degli articoli senza compromettere né lo spirito collaborativo dell'enciclopedia né il rigore scientifico degli studi storici?

Per cercare di capire lo stato degli argomenti di storia su Wikipedia si può partire dalla voce "Storia del Trentino"4. La pagina in questione è un esempio abbastanza evidente di problemi legati alla lettura storica su Wikipedia, in quanto presenta "buchi" di diversi secoli, "dimenticanze" strategiche ma, soprattutto, una visione parziale e strumentale nell'analisi dei fatti che hanno interessato questa terra di confine a partire dalla metà del XIX secolo. Un approfondimento sulla questione era già stato fatto da Tommaso Baldo nel suo articolo del 2015 «I “45 cavalieri" di Wikipedia, da chi e cosa è libera l'enciclopedia libera?" "5. Dando un'occhiata alla Cronologia ${ }^{6}$ della voce, inoltre, si nota come essa sia appannaggio di pochi utenti, i "soliti noti" a presidio dei contenuti, che di fatto creano un oligopolio attraverso il quale passa il vaglio di qualsiasi possibilità di modifica.

Il caposaldo principale di Wikipedia sono le fonti. L'enciclopedia online, infatti, si autodefinisce una fonte secondaria $\mathrm{o}$, più frequentemente, terziaria ${ }^{7}$, che riporta solamente informazioni già pubblicate altrove e generalmente accettate dalla comunità scientifica. Questa, di per sé, è un'ottima cosa, ma rende molto lento e frammentato il dibattito storico, soprattutto in uno strumento collaborativo e non specialistico come Wikipedia. Nello specifico della voce presa ad esempio, le proposte di modifica che riguardano argomenti minori o non propriamente canonici (ma che hanno iniziato ad essere accettati e divulgati grazie a più recenti studi storici) non vengono accettate e, peggio ancora, gli utenti proponenti vengono accusati di essere “austriacanti" (o fascisti/comunisti/nazionalisti/meridionalisti/etc., in base al tema).

Perché? Perché le nuove tesi, magari già accettate dagli esperti, vanno a cozzare con le fonti preesistenti: dalle enciclopedie cartacee ai sussidiari, dai siti web alla retorica nazionalista, la storiografia sedimentata crea un muro invalicabile di bibliografia, del tutto insensibile a qualsiasi discussione.

\footnotetext{
${ }^{2}$ «The top 500 sites on the web», in Alexa, URL: < http://www.alexa.com/topsites > [consultato il 7 febbraio 2017].

${ }^{3}$ "Top Sites in Italy», in Alexa, URL:< http://www.alexa.com/topsites/countries/IT > [consultato il 7 febbraio 2017].

${ }_{4}^{4}$ «Storia del Trentino», in it.wikipedia.org, URL: < https://it.wikipedia.org/wiki/Storia_del_Trentino > [consultato il 5 febbraio 2017]

${ }^{5}$ BALDO, Tommaso, «I "45 cavalieri di Wikipedia", da chi e da che cosa è libera l'enciclopedia libera?», in Giap, URL: < http://www.wumingfoundation.com/giap/?p=22562 > [consultato il 31 gennaio 2017].

${ }^{6}$ Cronologia della voce «Storia del Trentino», in it.wikipedia.org, URL:

< https://it.wikipedia.org/w/index.php?title=Storia_del_Trentino\&offset=\&limit=500\&action=history > [consultato il 7 febbraio 2017].

${ }^{7}$ «Wikipedia:Niente ricerche originali», in it.wikipedia.org, URL:

< https://it.wikipedia.org/wiki/Wikipedia:Niente_ricerche_originali > [consultato il 7 febbraio 2017].
} 
Il problema più importante è che il dibattito storico su Wikipedia è profondamente conservatore, frutto di una visione del passato ormai consolidata, dove molte proposte "nuove" vengono tacciate di revisionismo. Soluzioni? Bisogna convincere gli storici di professione a scrivere su Wikipedia, sia per innalzare il livello di qualità delle voci sia per superare il conservatorismo che contraddistingue l'enciclopedia libera.

Da molti anni Wikimedia Italia $^{8}$, corrispondente italiana della Wikimedia Foundation9 (fondazione no profit americana "proprietaria" di Wikipedia), sta intraprendendo la strada della collaborazione con le istituzioni GLAM ${ }^{10}$, acronimo formato dalle parole Galleries, Libraries, Archives e Museums. Dietro a queste sigle si raccolgono tutti i progetti, gli eventi, i corsi di formazione e gli accordi di vario genere che il "mondo Wikimedia" ha intrapreso con le istituzioni culturali per portare i professionisti su Wikipedia e contribuire ad innalzare progressivamente la qualità delle voci.

Le collaborazioni più immediate sono le edit-a-thon ${ }^{11}$, cioè maratone di modifica svolte nelle istituzioni culturali (musei e biblioteche in primis) con la partecipazione degli operatori della struttura. Questi eventi permettono di mettere in contatto i due mondi, quello dei wikipediani e quello dei professionisti del settore, ottenendo due risultati: da un lato i volontari dell'enciclopedia hanno la possibilità di affrontare argomenti specialistici in presenza di un corpus di fonti attendibili e con il supporto degli esperti e dall'altro gli operatori del museo hanno la possibilità di conoscere i meccanismi di Wikipedia direttamente da chi vi scrive, ricevendo di fatto una prima formazione.

Di eventi di questo tipo se ne svolgono da molti anni in tutto il mondo (al museo Soumaya di Città del Messico nel giugno del 2016 se n'è svolto uno di ben 72 ore ${ }^{12}$ !) ed anche in Italia si stanno diffondendo grazie all'interessamento sempre maggiore da parte delle istituzioni culturali; l'ultima maratona, dalla forte risonanza mediatica, si è svolta il 20 e il 21 gennaio 2017 presso la Galleria degli Uffizi di Firenze ${ }^{13}$.

Il livello di coinvolgimento tra "i progetti wiki" e le istituzioni culturali aumenta sensibilmente nel momento in cui viene avviato un progetto di Wikipediano in residenz $a^{14}$. Questa attività consiste

\footnotetext{
${ }^{8}$ Wikimedia Italia, URL: < http://www.wikimedia.it > [consultato il 8 febbraio 2017]

${ }^{9}$ Wikimedia Foundation, URL: < https://wikimediafoundation.org/wiki/Home > [consultato il 8 febbraio 2017].

${ }^{10}$ «GLAM», in Wikimedia Italia, URL: < http://www.wikimedia.it/glam > [consultato il 20 febbraio 2017].

11 «Edit-a-thon», in en.wikipedia.org, URL: < https://en.wikipedia.org/wiki/Edit-a-thon > [consultato il 19 febbraio 2017].

12 CRUZ Y CORRO, Andrés, LÓPEZ, María Fernanda, «Wikipedia edit-a-thon, 72 hours long, is recognized with a Guinness World Record», in blog.wikimedia.org, URL:

< https://blog.wikimedia.org/2016/07/22/mexico-soumaya-rodin > [consultato il 19 febbraio 2017].

13 «Editathon agli Uffizi», in it.wikipedia.org, URL:

< https://it.wikipedia.org/wiki/Wikipedia:Raduni/Editathon_agli_Uffizi > [consultato il 19 febbraio 2017].

14 «Wikipediano in residenza», in it.wikipedia.org, URL:

< https://it.wikipedia.org/wiki/Progetto:GLAM/Wikipediano_in_residenza > [consultato il 19 febbraio 2017].
} 
in un periodo di permanenza di un wikipediano presso la sede dell'ente in modo che possa essere avviata una profonda collaborazione. Il wikipediano ha innanzitutto il compito di "portare" su Wikipedia l'istituzione e le sue peculiarità, servendosi del materiale a disposizione come fonti per l'ampliamento di numerose voci correlate agli argomenti scientifici o umanistici che rappresentano la missione divulgativa della struttura. Altra attività importante che deve essere svolta dalla figura del wikipediano in residenza è quella della formazione interna, che può essere svolta in maniera reiterata ed approfondita e non solo "spot" come avviene con le maratone di scrittura. La completa collaborazione dell'istituzione e del suo personale è fondamentale per la buona riuscita della residenza, considerando anche la necessaria assistenza per tutte le possibili questioni legate ai diritti d'autore.

In Italia non mancano di sicuro i musei dedicati alla storia moderna e contemporanea, ma finora i contatti tra i due mondi sono stati sporadici. Le possibilità e le potenzialità, però, ci sono $\mathrm{e}$ sono evidenti. Le fondazioni storiche, inoltre, potrebbero essere dei partner molto preziosi in un altro settore nel quale Wikimedia Italia sta investendo una parte sensibile delle proprie risorse, e cioè quello delle scuole ${ }^{15}$.

I contatti con il mondo dell'istruzione si stanno facendo sempre più frequenti, con gli istituti scolastici che si dimostrano sempre più desiderosi di portare Wikipedia fra i propri banchi. In questo modo gli studenti hanno la possibilità di apprendere meglio il funzionamento di uno strumento che generalmente utilizzano in maniera passiva, facendo solo "copia-incolla" quando devono svolgere delle ricerche. L'obiettivo formativo finale di questo genere di corsi, però, non è tanto quello di creare nuove voci bensì quello di riuscire ad instillare la scintilla dell'analisi critica dei fatti e delle fonti: obiettivo, questo, che non è molto diverso da quello che dovrebbe animare il lavoro di ricerca di ogni buon storico.

Se il caposaldo di Wikipedia sono le fonti, è proprio in questo ambito che l'intervento degli storici sarebbe fondamentale per innalzare la qualità del dibattito. Un progetto parallelo ma molto importante è Wikisource ${ }^{16}$, la biblioteca digitale e multilingue contenente testi in pubblico dominio o con licenze libere. Esso è molto apprezzato dal mondo bibliotecario, ed infatti sono in corso numerosi progetti di digitalizzazione di libri ed immagini, soprattutto antichi; questo è il modo più diretto ed immediato per rendere disponibili le fonti per future ricerche e analisi.

Ciò che più conta, però, è che gli storici (come anche gli esperti di altre discipline) inizino finalmente a superare le proprie resistenze nei confronti di Wikipedia e che inizino a partecipare al suo sviluppo. Perché solo grazie a loro, e alle loro più recenti ricerche e pubblicazioni, sarà

\footnotetext{
${ }^{15}$ «Wikipedia va a scuola», in Wikimedia Italia, URL:

< http://www.wikimedia.it/cosa-facciamo/progetti-le-scuole > [consultato il 20 febbraio 2017].

16 «Wikisource in italiano», in it.wikisource.org, URL: < https://it.wikisource.org/wiki/Pagina_principale > [consultato il 20 febbraio 2017].
} 
possibile superare l'immobilismo che finora regna negli articoli di storia dell'enciclopedia online più famosa del mondo e per far sì che, almeno su Wikipedia, l'analisi storica possa contare su una visione più ampia dei fatti prescindendo dall'atavica contrapposizione tra vincitori e vinti. 


\section{L'AUTORE}

Cristian CENCI è laureato in Economia e Gestione dell'Ambiente e del Turismo presso l'Università degli Studi di Trento. Si avvicina al mondo di Wikipedia nel 2007; dal 2015 è membro dello staff di Wikimedia Italia in quanto project manager di Wiki Loves Monuments, concorso fotografico internazionale dedicato alla salvaguardia dei beni culturali. Come volontario dei progetti Wikimedia ricopre il ruolo di coordinatore regionale per il Trentino-Alto Adige/Südtirol.

URL: $<$ http://www.studistorici.com/progett/autori/\#Cenci > 